\title{
Passive infrared detector used for infrastructure protection
}

\author{
M. Kastek, H. Madura \& T. Sosnowski \\ Institute of Optoelectronics, Military University of Technology, \\ Warsaw, Poland
}

\begin{abstract}
The article presents passive infrared detectors (PIR) used for protection of large objects (buildings, airports, border) or large areas have larger detection ranges and complex algorithms of signal analysis, on which significantly depends efficiency of detectors operation. The construction and principle of operation of passive IR detectors (PIR detectors) of a large detection range is presented. The paper presents design and principle of operation of a passive IR detector of large detection range. Significant virtue of the described PIR detector is highly efficient detection of very slowly moving or crawling people.
\end{abstract}

Keywords: passive infrared detector, security systems, probability of detection.

\section{Introduction}

Passive infrared detectors (PIR) operating in far infrared range $(8 \div 14 \mu \mathrm{m})$ are frequently used in security systems for people detection. Because of their passive operation, it is difficult to detect them and due to suitably chosen spectral range of operation they are low sensitive to disturbances caused by sun radiation. For PIR detectors used in security systems, the pyroelectric sensors of high sensitivity are often used $[1,2]$. Passive infrared detectors (PIR) used for protection of large objects (buildings, airports) or large areas have larger detection ranges and complex algorithms of signal analysis, on which significantly depends efficiency of detectors operation.

Essential drawback of currently available PIR detectors is their low efficiency of detection of slowly moving or crawling people. Efficiency of slowly moving objects is low because the changes of thermal radiation from these objects are close to thermal fluctuations of a background [3]. Moreover, to detect slowly 
moving (or crawling people), the lower frequency limit of a transmission band of PIR detector have to be close to zero. When this condition is fulfilled, increase in low frequency noises is observed. Thus, detection algorithms must be different than these used for typical PIR detector. To detect crawling people, the higher number of sensors should be applied (more detection zones) what increases a signal-to-noise ratio because each of the sensors can "see" smaller observation area.

Multitude of factors influencing the signal received from a pyroelectric sensor often causes false alarms generated by PIR detectors. Therefore in order to reduce a number of false alarms caused by, e.g., air turbulences or changes in a background temperature resulting from sun radiation, the sensors with two active elements are used (two sensors) and alarm signal is determined by analysis of a difference of their output signals $[4,5]$. Distinguishing a useful signal (coming from an object) from a noise signal (arising as a result of disturbing factors) is a serious recognition problem. Thus, important task of PIR detector, especially of elongated detection zone is such conversion and analysis of the signal to achieve relatively high probability of object detection with simultaneous low probability of false alarm detection [6].

\section{Construction and principle of operation of PIR detector}

Main elements of PIR detector are the objective (mirror or refraction one), set of pyroelectric sensors, and electronic systems (Fig. 1). The sensors convert an optical signal, emitted from the "being observed" surface, into an electrical signal. This signal is processed in the electronic systems (it is amplified, filtered, sampled) and next analyzed in a microprocessor system.

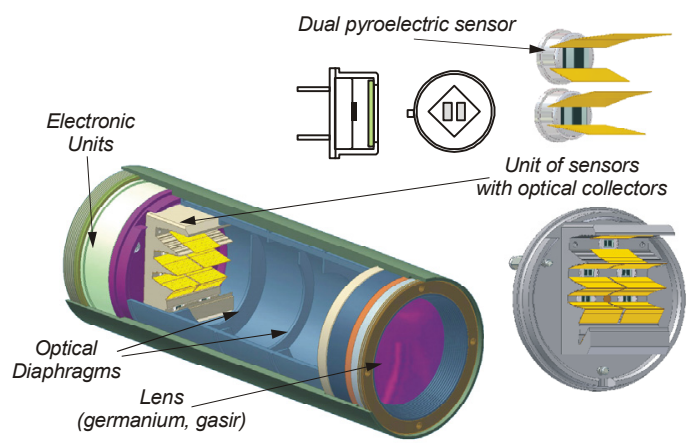

Figure 1: $\quad$ Simplified diagram of PIR detector and pyroelectric sensors with optical collectors.

The PIR detector presented here detects the crawling people at the distance of $140 \mathrm{~m}$. High signal-to-noise ratio was obtained due to application of the larger number of pyroelectric sensors, i.e., larger number of detection zones (channels). Application of a larger number of sensors forces the necessity to develop 
a complex optical system. The optical system of PIR detector has to ensure such a position of detection zones to avoid existence of the areas that "cannot be seen" by a sensor [3].

Thermal radiations generated by slowly moving and (especially) crawling people are characterized by similar amplitudes of luminance and velocities of this luminance changes in time such as fluctuation change of background radiation. The amplitude of a signal from pyroelectric sensors is directly proportional to the velocity of a change of a radiation signal in time (i.e., to the velocity of a moving object). Disadvantageous property of pyroelectric sensors is a voltage drift at the changes of ambient temperature (pyroelectric sensor is equipped with a field transistor playing a role of a voltage follower). Thus, to have a sensor efficiently detecting slowly moving people, it is necessary to develop an algorithm eliminating the changes of a signal from a sensor caused by background noise as well as the changes of a signal caused by temperature drift of a pyroelectric [4].

A simplified diagram of an electronic circuit of PIR detector is shown in Fig. 2. At irreversible input of the amplifying stage, a reference voltage is applied, obtained from a resistance divider and a digital potentiometer included between the supply voltage and the system's ground. The reference voltage (set by means of a digital electronic potentiometer which is controlled from a microprocessor) is used for elimination of a constant component of a signal coming from a pyroelectric sensor.

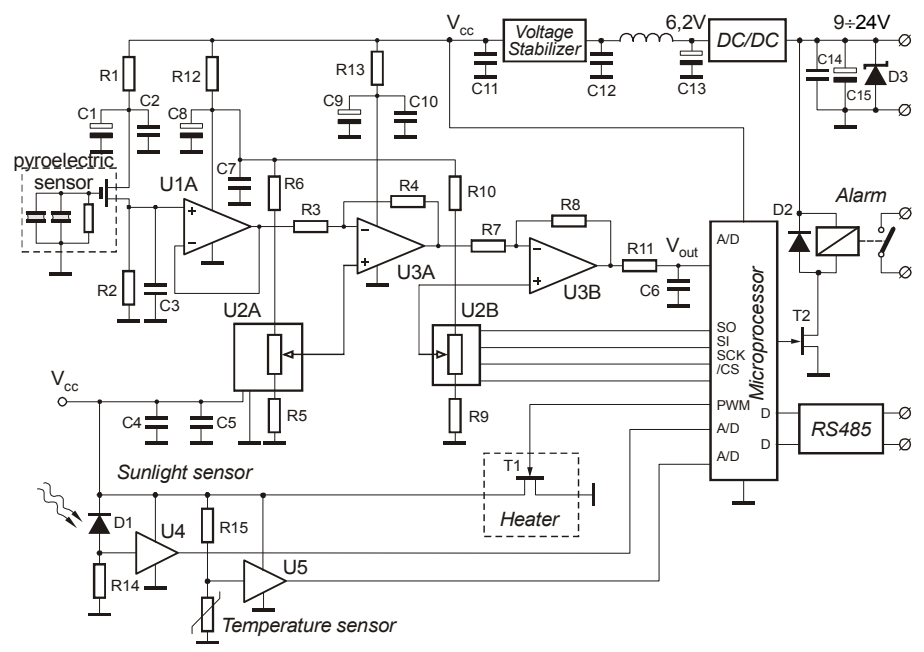

Figure 2: $\quad$ Simplified functional scheme of an electronic system of PIR detector.

This constant component significantly varies together with temperature changes occurring near a pyroelectric sensor (because of the change of output current a field transistor, dependent on temperature). The output voltage of an amplifier will be measured and analyzed by a microprocessor which in its 
operational algorithm will take into account the levels shifts (potentiometer settings). It is a real virtue of the performed system.

Application of electronic potentiometers requires determination of their working ranges by means of external resistors. In the PIR's electronic system, the system for measurement of sun radiation intensity was used. The information from this system is taken into account in an intruder detection algorithm. Moreover, a temperature sensor was used in the system. The data from a temperature measuring system can be applied to switch-on a miniature heater, mounted inside PIR detector housing. Small increase in a temperature of the PIR detector's inside (in relation to ambient temperature) protects deposition of water vapour on optical elements (lens, concentrator mirrors, and optical windows of sensors).

Passive IR sensors have specially formed angular characteristics of detection, achieved by application of appropriate optical elements, placement of PIR detectors on the focal plane (Fig. 1) and use of additional mirrors acting as beam concentrators [4].

The quality of optical design was verified by examining the relative irradiance of sensors by reflected radiation as a function of angle between mirror and detector surfaces. ZEMAX software makes it possible to calculate relative irradiance of seven sensors paired up with optical concentrators as a function of target movement in particular detection zones. The results were then verified against real measurement results performed on a laboratory test bed, in order to compare the expected values of parameters with those measured for a real device for all its detection zones.

\section{Method of analysis of PIR detector signal}

Detection of a person in a detection zone of PIR detector occurs when a signal level at the output of a detection system, caused by infrared radiation emitted by a person, exceeds a conventional detection threshold. To minimize a probability of false alarms, an adaptation detection threshold "following" the changing atmospheric conditions should be determined [4, 5, 7].

An analysis of the signal, caused by the moving person and background existing in a field of view of a detector, is made in a microprocessor system. The signal from a detection system is sampled and the voltages of the subsequent signal samples $S_{D}$ are added. Then, a value of such a signal determines Eq. (1)

$$
S_{\text {raw }}(i)=\sum_{j=i}^{i-N_{s}-1} S_{D}(j)
$$

where $S_{\text {raw }}(i)$ is the ith sample of the total signal and $N_{s}$ is the number of added samples.

This signal it is a numerical value determined by a microprocessor on the basis of the readout from analogue-digital converter and the set value of a digital potentiometer (Fig. 2), which was averaged for 20-ms period.

An average value of a signal for $N$ samples (Fig. 3) is determined according to the following equation 


$$
\bar{S}_{\text {raw }}(j)=\frac{1}{N} \sum_{i=j-N+1}^{j} S_{\text {raw }}(i)
$$

where $N$ is the number of samples needed for determination of the level of a constant component and $\bar{S}_{\text {raw }}(i)$ is the average value determined for the $i$-th moment (the $i_{t h}$ sample).

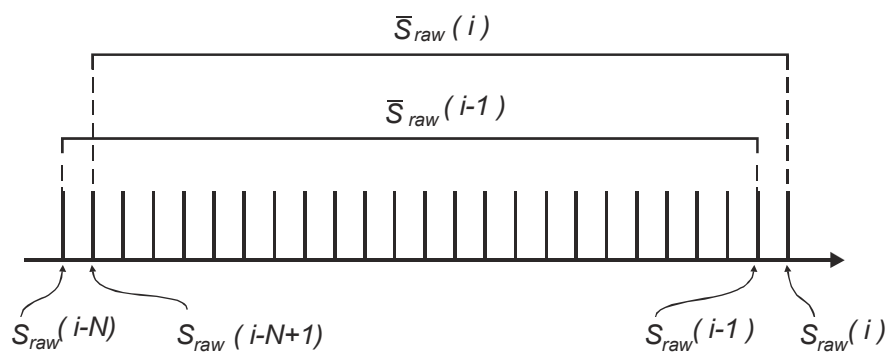

Figure 3: $\quad$ Notations used in a signal analysis method.

Instantaneous deviation from an average value (being an instantaneous level of a constant component) is determined as:

$$
R(i)=S_{\text {raw }}(i)-\bar{S}_{\text {raw }}(i) \text {. }
$$

A central moment of the first row for a set of the parameters $\left\{S_{\text {raw }}(i)\right\}$ can be determined as an instantaneous average value of deviations from the average from a relation:

$$
\bar{R}(i)=\frac{1}{C} \sum_{j=i-C+1}^{i} R(j)=\frac{1}{C} \sum_{j=i-C+1}^{i}\left[S_{\text {raw }}(j)-\bar{S}_{\text {raw }}(j)\right],
$$

where $C$ is the number of signal samples for which a correction is determined.

Next, a signal component is determined from the following equation:

$$
S(m)=\frac{1}{M} \sum_{i=m-M+1}^{m}\left|S_{\text {raw }}(i)-\bar{S}_{\text {raw }}(i)-\bar{R}(i)\right|,
$$

where $M$ is the number of samples in the process of averaging for the $k_{t h}$ set of algorithm parameters, while the average value $\bar{S}_{\text {raw }}$ is the local reference level and $\bar{R}$ is the correction resulting from the long-lasting tendency of a signal change.

Finally, the equation allowing for determination of a signal component takes a form:

$$
S(m)=\frac{1}{M} \sum_{i=m-M+1}^{m} \mid S_{\text {raw }}(i)-\frac{1}{N} \sum_{j=i-N+1}^{i} S_{\text {raw }}(j)-\frac{1}{C} \sum_{j=i-C+1}^{i}\left[S_{\text {raw }}(j)-S_{\text {raw }}(j)\right] .
$$

For particular case, when $N=C$, the above equation is

$$
S(m)=\frac{1}{M} \sum_{i=m-M+1}^{m}\left|S_{\text {raw }}(i)-\frac{2}{N} \sum_{j=i-N+1}^{i} S_{\text {raw }}(j)+\frac{1}{N^{2}} \sum_{j=i-2 N+1}^{i} S_{\text {raw }}(j)\right| .
$$

A signal for further analysis constitutes the weighted sum of signals calculated for each set of parameters according to the relation: 


$$
S(m)=\sum_{k=1}^{K} w_{k} S_{k}(m),
$$

where $w_{k}$ is the coefficient of a signal component.

A signal, subjected to the analysis referred to objects (intruders) detection, is determined from a relation

$$
S^{*}(m)=G \cdot S^{2}(m),
$$

where $G$ is the amplification (constant value) defined for each PIR detector.

A value of an alarm threshold is adaptively determined as:

$$
T_{k}=A \cdot \frac{1}{K} \sum_{i=k}^{K+k-1} S_{i}+B \cdot f\left(p_{S}\right)+C,
$$

where $A$ is the constant value, determining intensity of influence of previous samples of a signal on a value of a detection threshold, $K$ is the number of samples used for averaging process, $C$ is the parameter (constant) the value of which is dependent on the parameters that are set by a user, $B$ is the constant value determining the intensity of influence of sun lighting on the detection threshold value, and $f\left(p_{S}\right)$ is the function dependent on the level of the sun lighting $p_{S}$.

PIR detector generates the alarm signal when the amplitude of the signal read form a detector of the given detection zone (channel) is higher than adaptively determined alarm threshold. It means that alarm signal is generated by PIR detector in case when the following inequality is fulfilled

$$
T_{k}>S_{k}^{*} \text {. }
$$

One the most serious problems of PIR detector operation are to keep high detection probability at low probability of false alarm detection. At night, a level of thermal noises of a background is significantly lower that at the day. Thus, in IR sensor, switching IR sensor in day and night modes was used by applying the data from a sensor of a sun lighting level [7].

Passive PIR sensor was made as an element of security system of special, valuable object. It has seven detection zones without blind spots and sophisticated method of intruder detection, capable of finding slowly moving, or even crawling people. In order to test its operation and to assess the efficiency of implemented signal processing method, the measurements of various parameters were performed, both under laboratory conditions and during field tests.

\section{Result of investigations of method used in PIR detector}

During the measurements, carried out under various metrological conditions, the data were registered allowing for checking the correctness of algorithm operation, i.e., of a detection threshold determination made in a microprocessor system. The results of signals registration and the calculated values of detection thresholds were registered using the software developed for communication between PIR detector and computer. This software provides registration of signals from particular detection zones (channels) and registration of signals from particular stages of signal analysis. Figure 4 shows the test field of PIR detector. 

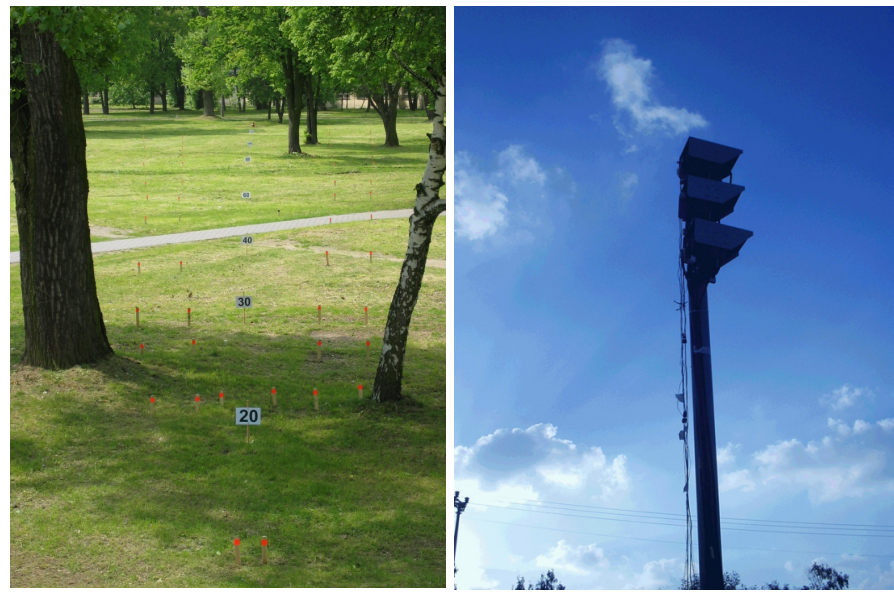

Figure 4: The test field and PIR detector during the tests.

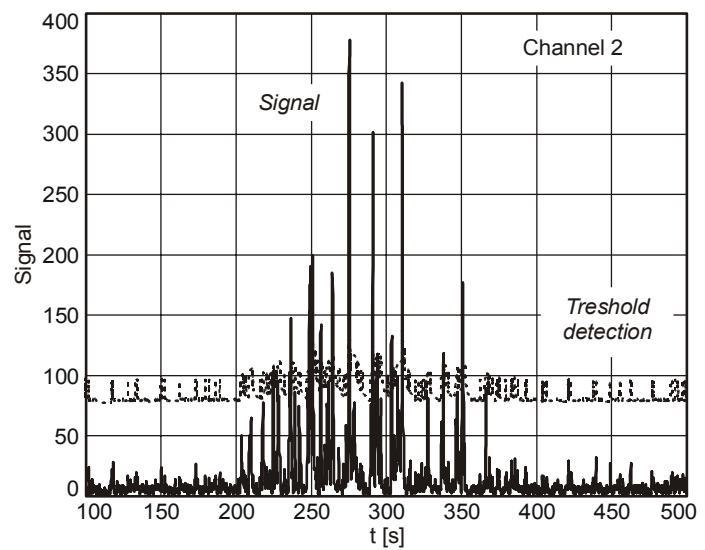

Figure 5: $\quad$ Signal in detection channel 2 of PIR detector (ambient temperature $26^{\circ} \mathrm{C}$, velocity of a crawling person $0.1 \mathrm{~m} / \mathrm{s}$, distance $140 \mathrm{~m}$ ).

Figure 5 present selected records of signals from a PIR detector which were produced due to a crawling person in an inspection zone. The presented results illustrate a change of a signal in the detector channels caused by a crawling person and adaptive change of a detection threshold resulting from the change of the analyzed signal.

The investigation results presented in the above figures confirmed proper operation of a detector, especially correctness and high efficiency of signal analysis method. Figure 5 shows a detection threshold following thermal changes of a background which, for this measuring case, were caused by sun radiation.

Figure 6 presents the recorded changes of a signal in detection channel 1 of PIR detector caused by a person walking with a velocity of $1 \mathrm{~m} / \mathrm{s}$, what proves universality of the used algorithm of a signal analysis allowing for detection of very slowly or very quickly moving people. 


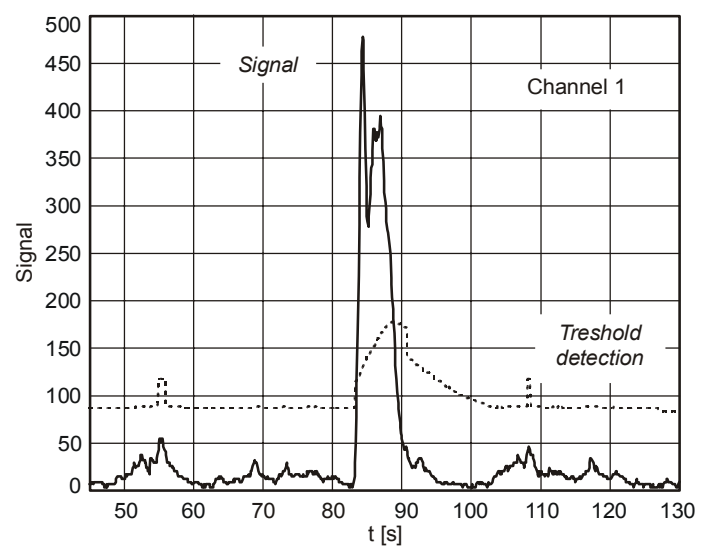

Figure 6: $\quad$ Signal in detection channel 2 of PIR detector registered at night (ambient temperature $25^{\circ} \mathrm{C}$, velocity of a moving person $1 \mathrm{~m} / \mathrm{s}$, distance $140 \mathrm{~m})$.

Efficiency of a signal analysis method, the aim of which is to determine a threshold value of a signal allowing for object detection, can be confirmed by adequately high probability of object detection and low probability of false alarm.

\section{Determination of a person detection probability}

A signal coming from an object is disturbed with the noise generated by a background and electronic systems of PIR detector. It can be assumed that the noise at the detector output is a white noise, while instantaneous values of voltages can be described with a normal distribution with the expected value $\overline{x_{N}}$ equal to zero and the standard deviation $\sigma_{N}$.

For a signal with Gaussian distribution of probability density of instantaneous values of voltages, the detection probability is described from a relation

$$
P D=1-\frac{1}{\sigma_{N} \sqrt{2 \pi}} \int_{x_{D}^{-x_{S}}}^{\infty} \exp \left(\frac{-x^{2}}{2 \sigma_{N}^{2}}\right) d x,
$$

where $x_{D}$ is the value of a detection threshold and $\bar{x}_{S}$ is the average value of the signal.

Under the same conditions, the probability of a false alarm is expressed as

$$
P F A=\frac{1}{\sigma_{N} \sqrt{2 \pi}} \int_{x_{D}}^{\infty} \exp \left(\frac{-x^{2}}{2 \sigma_{N}^{2}}\right) d x .
$$

For low probability of false alarms and high probability of object detection when it is fulfilled, Eqs. (12) and (13) can be transformed into the following form

$$
P D=1-\frac{\sigma_{N}}{\sqrt{2 \pi\left(x_{S}-x_{D}\right)}} \exp \left(\frac{-\left(x_{S}-x_{D}\right)^{2}}{2 \sigma_{N}^{2}}\right),
$$




$$
P F A=\frac{\sigma_{N}}{\sqrt{2 \pi} x_{D}} \exp \left(\frac{-x_{D}{ }^{2}}{2 \sigma_{N}^{2}}\right) .
$$

These probabilities depend on two parameters, the ratio of a detection threshold value to the standard noise deviation $x_{D} / \sigma_{N}$ as well as the ratio of an average value of a signal to the standard noise deviation $\bar{x}_{S} / \sigma_{N}$.

The detection probability can be presented as a function of signal-to-noise ratio by introducing the notation $S N R=\frac{\bar{x}_{S}}{\sigma_{N}}$ and transforming Eq. (15) we have:

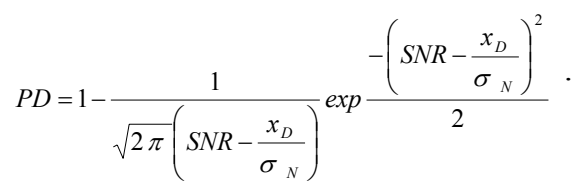

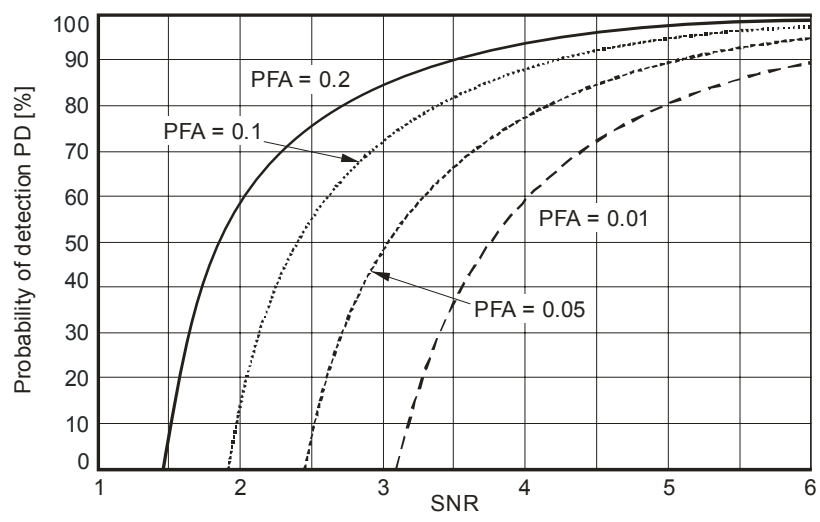

Figure 7: Probability of object detection for signal processed with the proposed analysis method.

For calculations of detection and false alarm probability, a standard noise deviation should be determined basing on the changes of the $S_{k}(m)$ parameter, and a threshold value should be substituted instead of the $x_{D}$ value.

Figure 7 presents the diagrams of detection probability as a function of the signal-to-noise ratio for a few assumed probabilities of a false alarm. According to Eq. (16), the probability of false alarm depends on the $x_{D} / \sigma_{N}$ ratio. In a method of signal analysis used for PIR detector, one can take the higher value of $x_{D}$ (higher $S N R$ ). After filtration, also the lower value of $\sigma_{N}$ is obtained what ensures lower values of false alarm probability.

\section{Conclusions}

To recapitulate, it should be stated that the developed PIR detector detecting slowly moving and crawling people has the parameters allowing for protection of 
large objects (buildings, airports, border) or large areas, its application in security systems. Satisfactory results of laboratory and field investigations of the developed and performed model of a PIR detector have confirmed that the developed detector detects the objects moving with the velocities from $5 \mathrm{~cm} / \mathrm{s}$ to $5 \mathrm{~m} / \mathrm{s}$. A detection range of crawling people (for the performed model) is of the order of 140-150 m. In a method of signal analysis used for PIR detector, gives lower values of false alarm probability. The new method of signal processing gives a higher the detection probability than PIR with normal signal processing.

\section{References}

[1] Feng C. and Xu P., The detection mechanism of LiTaO3 type II pyroelectric detectors. III. The total pyroelectric effects, Infrared Phys. Techn. 40, pp. 79-82, 1999.

[2] Yoshiike N., Morinaka K., Hashimoto K., Kawaguri M., Tanaka S., 360 direction type human information sensor, Sensor and Actuators, vol. 77, pp. 199-208, (1999).

[3] Kastek, M., Sosnowski, T., Polakowski, H., H., Dąbrowski, M., Orżanowski, T., Long-range PIR detector used for detection of crawling people, Proc. of SPIE Vol. 7113, 71131F, 2008.

[4] Kastek M., Sosnowski T, Piątkowski T., Passive infrared detector used for detection of very slowly moving of crawling people, Opto-Electronics Review 16 (3), pp. 328-335, 2008.

[5] Madura H., Method of signal processing in passive infrared detectors for security systems, WIT Transactions on Modeling and Simulation 46, pp. 757-768, 2007.

[6] Kastek, M., Madura, H., Morawski M., Piątkowski T., Powiada E., Polakowski H., Test bed for measurement of angular parameters of passive infrared sensors, Infrared Phys. Techn. 49 (3 SPEC. ISS.), pp. 198-201, 2007.

[7] Sosnowski, T., Madura, H., Kastek, M., Piątkowski T., Powiada E., Method of objects detection employing passive IR detectors for security systems, Proc. of SPIE Vol. 7113, 71131G, 2008. 